\title{
Les pratiques spatiales des requérants d'asile dans les abris de la protection civile de Genève
}

\section{Karim Mouzoune, Genève}

Face aux crises et aux conflits internationaux, aux catastrophes naturelles et technologiques et aux guerres, aux tensions entre l'Est et l'Ouest dues à la guerre froide, à la course aux armements, aux clivages sans cesse croissants entre nations pauvres et nations riches, la Suisse a pris des mesures préventives destinées à protéger sa population en cas de menace. C'est ainsi qu'elle a créé, dans les années soixante-dix, des abris pour protéger les civils. L'objectif est d'offrir à chaque habitant un espace le protégeant des effets des armes, des rayonnements ionisants et de la contamination par des substances chimiques dangereuses grâce à des murs épais en béton armé. Ces abris sont conçus et aménagés de manière à y permettre, au besoin, un séjour prolongé. Mais depuis leur création, à défaut de crise majeure, les abris ont servi à loger des groupes artistiques, comme des fanfares en visite à Genève.

A partir d'octobre 1998 et face à l'arrivée massive de requérants d'asile en provenance essentiellement du Kosovo, l'AGECAS (l'Association Genevoise des Centres d'Accueil pour Candidats à l'Asile) s'est vue contrainte d'ouvrir des abris de la protection civile afin d'héberger les nouveaux arrivants à Genève. Ces espaces deviennent pour les requérants des lieux d'habitation et d'ancrage (FISCHER 1981), des coquilles personnelles (MOLEs \& RoHmer 1972) qu'ils ont délimitées, possédées, notamment par des pratiques subtiles de réaménagement.

L'objet de cette étude est d'analyser les territorialités psychosociologiques des requérants d'asile dans les abris de la protection civile et leurs conséquences sur les relations interpersonnelles. Emprunté à G.-N. Fischer, le concept de territorialités psychosociologiques s'exprime à travers les interactions hommeenvironnement, en particulier lors de la nidification de l'espace vécu. La nidification est définie comme une conduite permettant à chacun de créer un «chez soi» grâce à son marquage, à sa délimitation, à la familiarisation avec ses différentes composantes, à la construction d'un lieu personnel comme forme d'enracinement, notamment par l'injection de significations privées dans un espace impersonnel (FISCHER 1981).

\section{Eléments généraux de l'enquête}

Nous avons effectué une enquête dans les abris de la protection civile de Carouge et d'Annevelle dont les capacités d'accueil sont respectivement de 80 et 120 places. Notons que les capacités réellement utilisées sont de 50 et 120 places. Cette enquête, qui a duré de mars à août 1999, a porté sur 50 personnes dans l'abri de Carouge et 40 dans celui d'Annevelle.

\subsection{Aperçu sur la population enquêtée}

Les requérants d'asile ont fui la guerre, les conflits interethniques, le terrorisme et les persécutions politiques. Dans la communauté des requérants kosovars, $70 \%$ de personnes sont des ruraux chassés de leurs villages par les troupes serbes; les $30 \%$ restant viennent de villes comme Prishtina, Serizaj et Gjilan. Les demandeurs d'asile africains et irakiens sont, pour la plupart, issus de milieux urbains. Si les Kosovars ont transité par l'Italie pour arriver en Suisse, les Africains par contre sont passés par la France.

\subsection{Modalités de l'enquête}

Partant d'une enquête par interview sur 90 personnes dont 20 femmes, et de l'observation directe du vécu dans deux abris, nous traitons la manière dont ils sont occupés, réaménagés, acceptés ou rejetés, et la façon dont les rapports interpersonnels y sont organisés. Il s'agit donc de l'étude des différentes territorialités psychosociologiques (les relations hommeespace-temps) situées au niveau horizontal d'un lieu dépourvu, au départ, d'espace d'intimité.

L'échantillon est composé à $80 \%$ de requérants kosovars; les $20 \%$ restant viennent d'Afrique (Algérie, Tunisie, Sénégal, Burkina Faso, Togo, Guinée Bissau et Sierra Leone) et du Proche-Orient (Irak). Chez les hommes, $80 \%$ des personnes interrogées sont âgées entre 17 et 35 ans; l'âge des $20 \%$ restant varie entre 36 et 65 ans. Chez les femmes, 90\% d'interviewées sont âgées de moins de 45 ans; l'âge des $10 \%$ restant se situe entre 46 et 60 ans. 10 familles composées de 40 personnes ont fait l'objet d'observations. Aucune d'elles n'est monoparentale, mais elles regroupent souvent des ascendants (grands-parents) et des proches (oncles et tantes).

\subsection{Questionnaire}

Le questionnaire utilisé dans nos entretiens se focalise sur les interrogations suivantes:

- Depuis combien de temps habitez-vous dans l'abri?

- Que représente-t-il pour vous?

- Vous y sentez-vous en sécurité?

- Comment l'aménagez-vous?

- Comment vous y organisez-vous?

- Y conservez-vous une certaine intimité? 
- Y a-t-il un contrôle de son accès?

- Essayez-vous de vous démarquer de vos voisins et par quel moyen?

- Comment sont vos relations avec vos voisins?

- Quels sont les éléments qui vous gênent dans l'abri?

- Quelles sont les qualités qu'il dégage?

- Quels sont les facteurs de tension qui s'y manifestent?

- Comment les gérez-vous?

- Y imposez-vous des interdits et lesquels?

Ces questions standards, traduites en albanais pour les Kosovars, sont complétées par d'autres suscitées par la discussion, notamment avec les femmes et les jeunes.

\section{Les pratiques de transformation de l'espace abri}

Très souvent vide, ou peu occupé, l'abri de la protection civile est un lieu qui connaît une forte pression humaine depuis l'arrivée des requérants d'asile. Le rapport à cet espace tient compte de l'enfermement, de l'entassement et du manque d'intimité. Sous cet angle, sa transformation correspond à la règle de l'efficacité: essayer d'optimiser l'utilisation des locaux afin de cohabiter avec le maximum de personnes. Toutefois, si des normes collectives (les règlements) qui déterminent son usage ou ses conditions d'accessibilité sont établies par les responsables de l'AGECAS et de la Protection Civile, des pratiques individuelles, issues des familles, viennent s'y ajouter. Elles rendent l'abri plus compartimenté, plus ou moins découpé par des marqueurs artificiels prégnants. Derrière tout acte, il y a un vécu. C'est ce que nous nous proposons d'exposer à travers cette analyse axée sur l'observation directe des pratiques de transformation de l'abri.

\subsection{La structure de l'abri}

L'abri est un sous-sol transformé en un refuge accueillant les requérants d'asile. Il représente un espace vécu pour des dizaines de familles et célibataires obligés d'y résider à défaut de places dans les foyers en surface. Pour Carouge, il s'agit d'un espace composé d'un réfectoire, d'une cuisine, de dortoirs de 96 places, de sanitaires et de bureaux des responsables de l'AGECAS et de la Protection Civile. Les dortoirs sont dotés d'armoires casiers qui les divisent en deux parties empêchant ses occupants de vivre une forte promiscuité.

L'abri d'Annevelle est spacieux en ce qui concerne le réfectoire, mais ses dortoirs de 270 places sont petits et longs, ce qui génère une forte promiscuité. Les résidents se sentent plus à l'aise dans le réfectoire que dans les dortoirs, justement à cause de la dimension, hélas restreinte, de cet espace synonyme de convivia- lité, car les groupes communautaires se réunissent les uns à côté des autres, mais non avec les autres ou en congruence avec eux.

Généralement, les abris de Carouge et d'Annevelle sont utilisés collectivement et sans séparation par l'ensemble des résidents qui s'y installent, sauf pour les sanitaires dont les uns sont réservés aux femmes et les autres aux hommes. Ils sont délimités, circonscrits par des frontières apparentes par rapport à l'extériorité. Les portes, même si elles restent ouvertes jusqu'à 24 heures, jouent le rôle de protection. Elles évoquent pour ceux qui vivent dans les abris l'idée d'espaces familiers, mais dont ils ne sont pas réellement les maîtres. Elles marquent aussi l'opposition dehorsdedans: il n'y a passage que s'il y a séparation. L'entrée désigne le rapport appartenance-non appartenance. Introduisant une distinction entre espace autorisé et espace non autorisé, les portes opèrent un filtrage symbolisé par la contrainte qui oblige les non-résidents à signaler leur présence dans les locaux aux responsables de l'AGECAS et de la Protection Civile.

Dans les abris enquêtés, une fois le seuil franchi, nous nous trouvons au sein d'un vaste lieu composé d'espaces fumeurs, de réfectoires, de dortoirs de célibataires et de familles et d'espaces auxiliaires réservés aux sanitaires. Les bureaux sont situés en retrait, empêchant tout contrôle direct des mouvements d'entrées-sorties dans les abris.

\subsection{Conduites collectives d'aménagement et esthétique fonctionnelle de l'espace clos}

Dans l'espace abri, draps et meubles sont des artifices utilisés par $90 \%$ de familles pour signifier la division familiale de l'espace clos et imprégner sa structure d'un semblant d'intimité. Les occupants, ayant l'impression de vivre "à découvert», sont obligés de se cacher (du regard) des autres. Les marqueurs spatiaux remplissent donc le rôle d'isolation visuelle, l'une des fonctions du mur selon A. Moles et E. Rohmer (1972: 47). Les marqueurs apportent, comme le souligne A. Bailly citant MC CanNel (1986), «des éléments explicatifs dans la construction d'une architectonique sociale»; ils mettent aussi en exergue l'importance de la relation entre l'information et le lieu, qui devient un véritable signifié.

Les efforts de transformation portent sur l'agrément du local, comme par exemple l'installation de meubles récupérés de la rue. Il s'agit de rendre le lieu plus agréable et, par là-même, accroître la convivialité. Des endroits leur sont prévus; c'est généralement dans les coins, à l'écart des matelas signifiant l'espace de repos (Photo 1).

Pensant quitter les abris de la protection civile pour être logées dans des appartements, les familles ont essayé de préparer leur ameublement en accumulant meubles, réfrigérateurs, machines à laver et postes de télévision; ces objets seront amenés au pays lors du retour. 


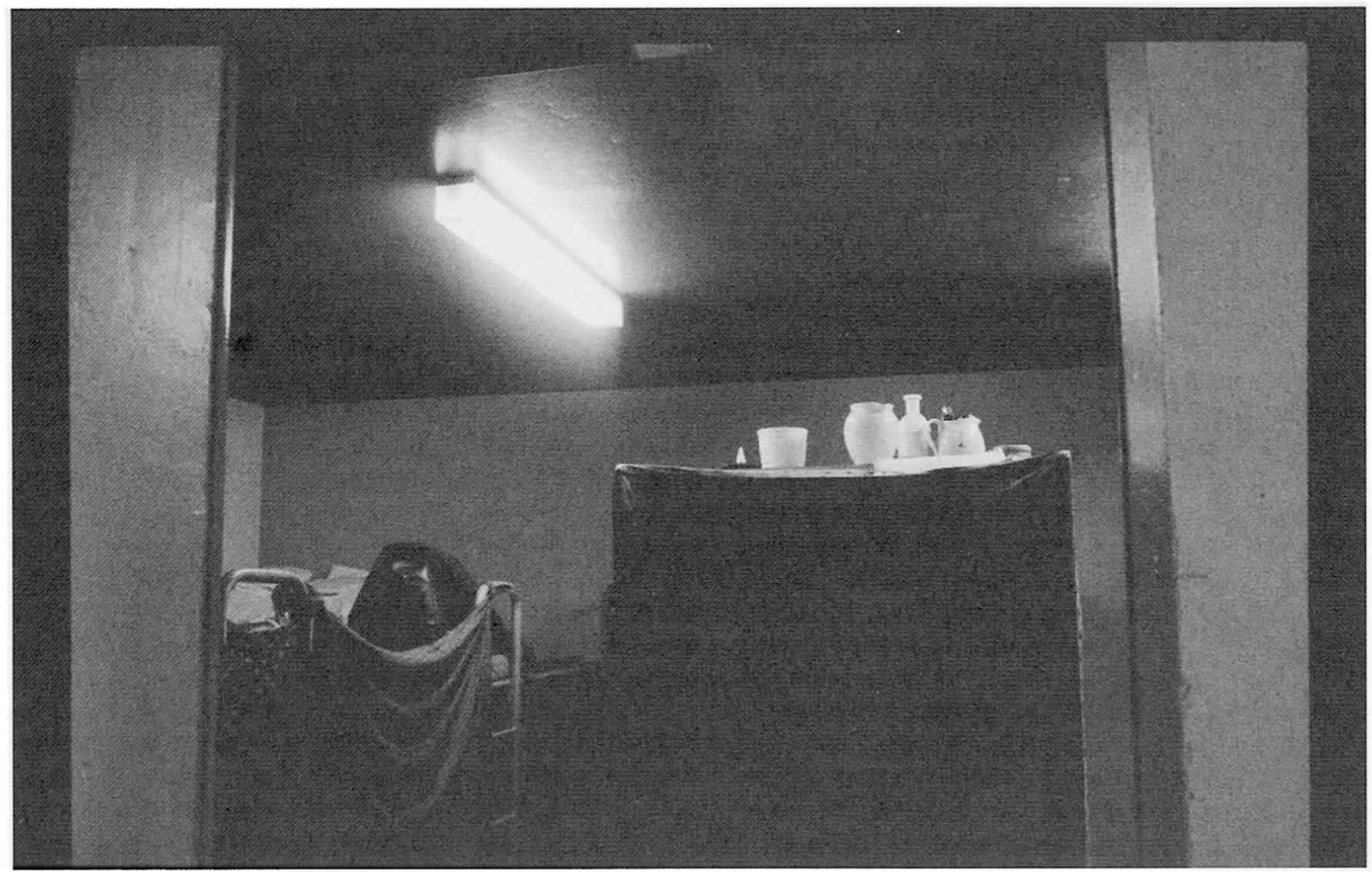

Photo 1: Dortoir des familles de la PC de Carouge

Photo: K. Mouzoune

The family quarters of the Carouge shelter

Familien-Schlafstelle im Zivilschutzraum von Carouge

Ainsi, à Carouge, l'abri de la protection civile prend progressivement le visage d'une «esthétique» nécessaire, qui par sa dimension «décorative», élimine les sentiments de répulsion à l'égard de l'espace clos chez seulement $15 \%$ de familles et $20 \%$ de célibataires. Dans l'abri, il existe également un «espace récréatif» utilisé par les adultes. Ce lieu joue le rôle de point de rencontre où on joue aux cartes, aux échecs, aux fléchettes, on discute des événements extérieurs - surtout du pays - et intérieurs, on écoute de la musique populaire nationale (Photo 2).

Sachant que leur séjour dans les abris de la protection civile peut être long (de 8 à 12 mois), les requérants ont organisé leur vie en communauté, renonçant souvent à certaines habitudes, comme par exemple l'usage tardif de la télévision ou le règlement des problèmes du volume des postes de radio et de télévision. Ils ont également instauré des interdits afin d'empêcher l'exacerbation des conflits: interdiction de fumer dans les dortoirs, d'augmenter le volume de la télévision ou de la radio à partir d'une certaine heure, voire de l'usage de ces appareils après minuit, de rentrer dans l'abri après minuit. Ce sont des interdits émanant des responsables de l'AGECAS et de la Protection Civile.
L'analyse du processus de transformation de l'abri montre comment cet espace peut être concrètement l'objet d'aménagements et de négociations, et qu'en outre, c'est à la fois le sentiment de maîtriser l'espace et la relation de chacun avec le système socio-spatial qui sont recherchés.

\subsection{L'abri: aménagement individuel et marquage spatial}

Les contraintes de l'entassement ont amené toutes les familles de la protection civile de Carouge à réaménager leurs petits espaces d'installation, faisant apparaître le fait que l'absence totale d'intimité, le non-respect de l'exigence spatiale d'un cadre familier, ou les problèmes de communication, peuvent augmenter les tensions et créer une charge mentale particulière. Ce réaménagement n'a perturbé ni la structure, ni l'organisation de l'abri, mais a exprimé le désir d'emprise, d'appropriation des lieux, de disposition d'une coquille personnelle bien que transparente et perméable. Précisons que les pratiques de réaménagement se sont déroulées dans les dortoirs occupés par peu de familles (ne dépassant pas le nombre de quatre) de faible taille (entre trois et quatre personnes). Ceci 


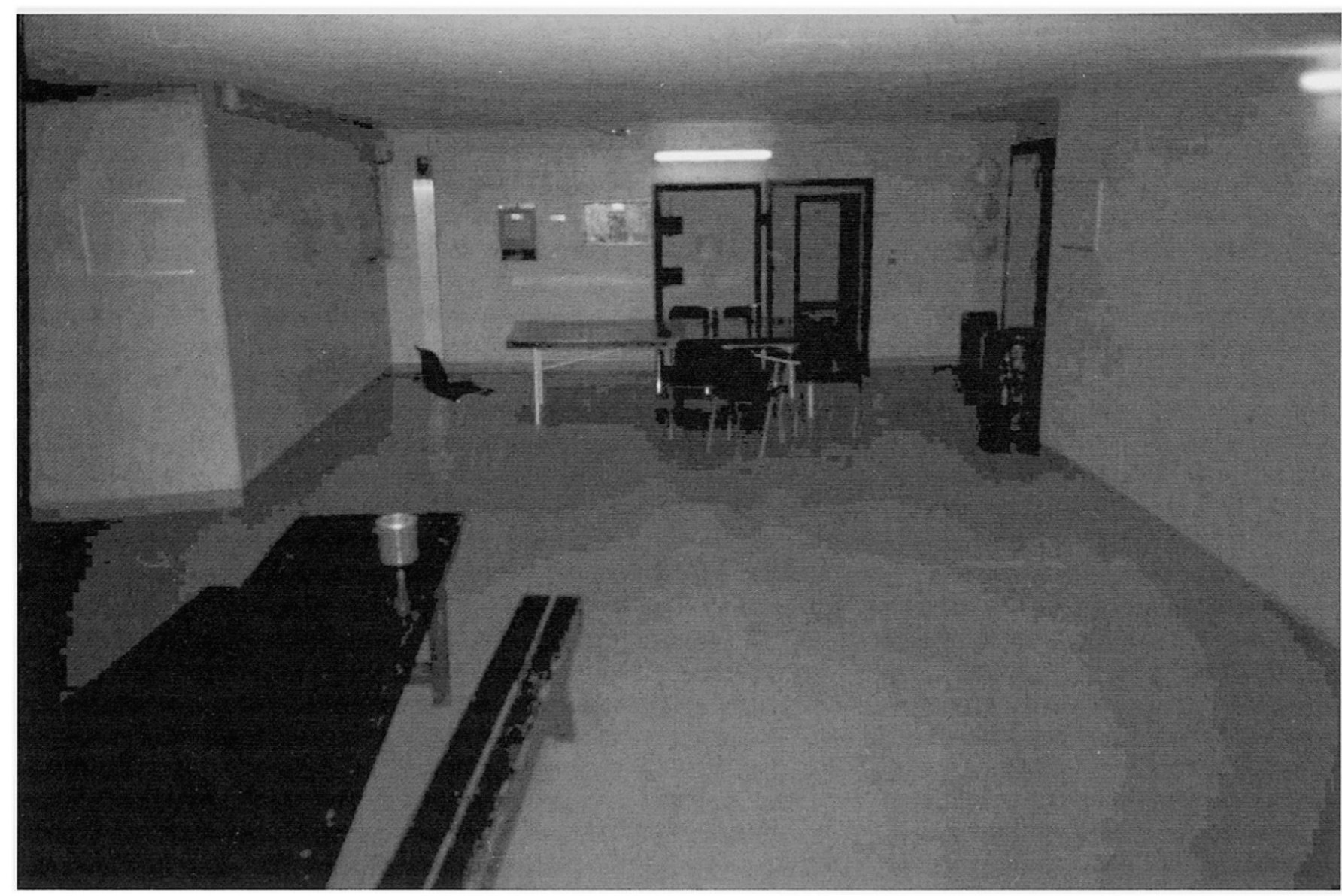

Photo 2: L'espace récréatif dans la PC d'Annevelle

Photo: K. Mouzoune The recreational room of the Annevelle shelter

Der Aufenthaltsraum in der Zivilschutzanlage von Annevelle

a beaucoup facilité la réalisation d'un «chez soi» sans heurts avec les voisins, ni bouleversements profonds dans l'organisation fonctionnelle spatiale commune. De telles pratiques revêtent plusieurs caractères:

- la répartition de la surface - non affectée à l'usage collectif - entre les différentes familles en fonction de leur taille;

- le marquage et la délimitation du territoire: chaque famille peut marquer son espace par un repère particulier afin d'y contrôler l'accès. Il s'agit d'un véritable bornage symbolique usant des marqueurs: meubles ou draps. On essaie de créer des obstacles pour limiter l'intrusion tant physique que visuelle de l'autre;

- la construction d'un espace personnel:elle s'exprime par l'injection de significations privées (habits, sacs) sur un espace impersonnel (Photo 3).

L'aménagement se traduit également par l'usage du mobilier privé chez $60 \%$ de familles et $10 \%$ de célibataires (armoires, buffets, réfrigérateur), ce qui donne l'impression d'être dans une pièce. Ce type de réorganisation spatiale répond à l'aspiration de retrouver une certaine intimité, sans ignorer les contraintes pesantes de la vie communautaire. Le plus frappant dans les abris reste l'aménagement de l'intérieur. Borné par divers artifices, chaque sous-espace provoque ce sentiment de se trouver dans une pièce équipée, dotée d'un confort moyen.

L'effort d'esthétisation rend le sous-espace moins austère, plus agréable, fonctionnellement plus humanisé. Cette esthétisation n'est pas sans générer des inconvénients, notamment le soir, au moment où tout le monde se trouve «chez soi». En effet, la promiscuité sur un fond sonore en provenance des postes de radio, allumés ici et là, dérange surtout les familles ayant des enfants en bas âge. En l'absence de confort acoustique même rudimentaire, le bruit stresse davantage les femmes et irrite les enfants. L'inconfort sonore renvoie à un signal d'intrusion dans l'intimité, à une agression de soi; d'où des réactions diverses qui vont de la complainte chez $85 \%$ de résidents, aux altercations avec ceux qui en sont à l'origine chez les $15 \%$ restant. Cependant, l'obligation de vivre en commun, les menaces d'expulsion venant des responsables amènent les individus à plus de raison; le bruit cesse et les 


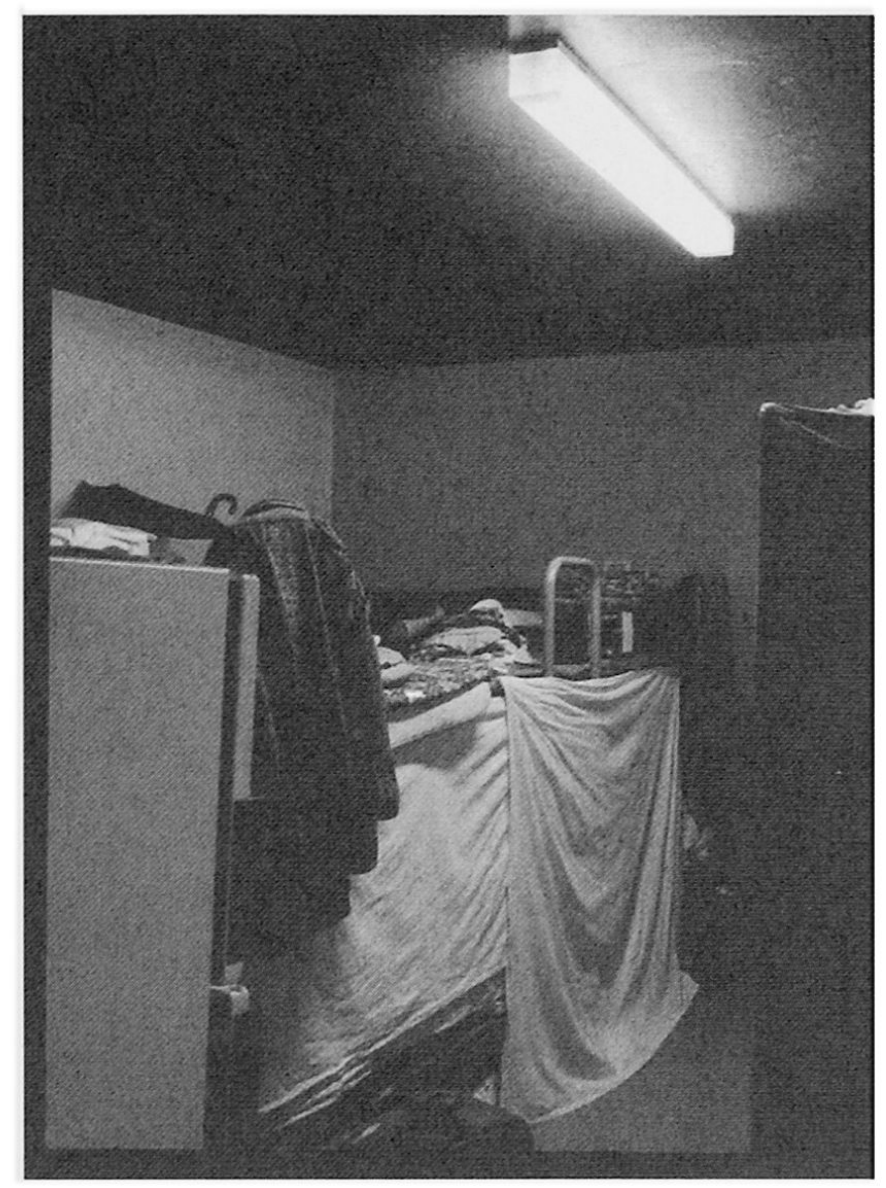

Photo 3: Réservation et appropriation d'un coin par une famille dans la PC de Carouge

A family has reserved and clearly marked off their sleeping area in the Carouge shelter

Die räumliche Abgrenzung einer Familien-Schlafstelle im Zivilschutzraum von Carouge

Photo: K. Mouzoune

tensions s'apaisent. Malheureusement, les dérapages sonores sont sporadiques, ce qui déclenche de nouvelles négociations sur fond de menaces d'exclusion.

Les pratiques de réaménagement ne sont pas dissociées de l'environnement social dans lequel vit chaque usager de l'abri. C'est pourquoi elles reflètent des rapports individu-groupe social-espace abri qu'il convient d'étudier.

\section{De l'enracinement à l'appropriation des sous-espaces abri}

L'organisation de l'abri permet d'entrevoir que cet espace constitue un enjeu; il accueille des pratiques, créant des conflits tantôt diffus, tantôt centrés. C'est de cette réalité observée que nous partirons pour analyser le rapport quotidien à l'abri et montrer qu'il forme, selon ses occupants, un lieu, ou des sous-lieux, que les individus identifient et auquel ils s'attachent. L'espace clos apparaît comme la sphère ressentie d'un enracinement; celui-ci reflète l'attitude d'implication de l'usager par rapport au lieu dans lequel il est fixé. Nous évoquons par là le fait que l'individu éprouve des sentiments, invente des façons de faire, réagit, se crée une forme d'adaptation malgré la contrainte que représentent l'entassement et l'enfermement. En somme, l'individu vit normalement la réalité abri, même si elle est provisoire. A travers un ensemble de relations, il la perçoit différemment suivant qu'elle est bonne ou mauvaise, positive ou négative. Ainsi, nous avons opéré la distinction entre deux types d'espace: un espace refusé qui traduit l'enchaînement à un lieu fermé, étroit, étouffant, stressant, et un espace accepté, c'est-à-dire un endroit sur lequel les individus ont l'impression d'étendre leur emprise.

L'idée d'appropriation permet de mieux rendre compte des différents phénomènes d'emprise sur le lieu abri. Elle éclaire de ce fait tout un aspect de la vie sociale. L'appropriation désigne globalement l'acte de prendre quelque chose pour soi, ou l'acte de rendre quelque chose propre à son usage. En un mot, s'approprier veut dire faire sien, s'attribuer, se donner la propriété de quelque chose, même si elle ne vous appartient pas (Fischer 1983: 40). Par l'appropriation s'opèrent une structuration de l'espace et une redisposition des choses ou des objets (FISCHER 1983: 40). A l'aide de différents artifices (signes, symboles), un individu (ou un groupe) indique qu'il contrôle l'espace. Cette mainmise est régie, dans le cas de l'abri à faible densité avec possibilité de créer son espace propre, par différents critères:

- la clôture résultant de l'initiative personnelle d'aménagement qui favorise certains replis, certains enfermements, certaines dissimulations;

- la capacité de modification personnelle d'un espace;

- la distance par rapport aux autres (membres de la famille, voisins);

- le degré d'attachement à un lieu et la possibilité de s'y identifier par le marquage et le réaménagement.

Grâce à cette emprise, chacun crée sa coquille, son espace personnel limité, différencié par le type et le nombre d'objets ou d'éléments qui s'y accumulent. «L'appropriation relève de la fonction d'entassement» (Fischer 1981: 94); elle revêt un double caractère. Le premier est bien connu chez les éthologistes (CALHOUN 1962, LORENZ 1970): je m'approprie un espace d'autant plus que j'y ai mis plusieurs objets. Le second a trait à l'entassement à plusieurs dans un même lieu. Suite à ses transformations, l'abri est vécu dans une large mesure à travers les restrictions matérielles et physiques, et les accommodements pratiques qu'il impose. Il est également vécu comme la somme des moindres recoins côtoyés, des gestes effectués et du 
temps écoulé. La part spatiale et spatio-temporelle de la culture de chaque requérant a guidé ses usages des lieux et ses rapports aux autres. Ici, connaissances savantes et sens commun éclairent, comme le souligne FerRIER (1998: 256), la manière d'habiter et suggèrent les principes qui en amélioreraient l'usage.

Par ailleurs, les relations avec l'autre et au lieu ont souvent fait de l'abri un lieu de ruptures organisationnelles, d'anicroches et de dissonances entre ses occupants. Les limites se dressent, les contraintes investissent les relations socio-spatiales. Plusieurs conflits sont apparus. Certains se sont déclenchés au sein de la communauté kosovare entre célibataires et familles à cause du bruit, ou entre familles d'origine rurale et d'autres issues de milieux urbains. Les citadines considèrent les rurales comme sales, voleuses, et leurs enfants mal élevés. Ces conflits, bien que réglés, laissent des traces au niveau des rapports interpersonnels, puisque les célibataires continuent de vivre en groupes compacts n'ayant pas de relations avec les familles; en même temps, les familles citadines se distancent des rurales, évitant au maximum de les croiser dans les lieux communs. Ces relations dissymétriques se reflètent dans l'usage de certains lieux comme la cuisine. Cette dernière n'est investie par les célibataires que lorsque que les familles ont terminé de cuisiner. Les familles issues de milieux urbains utilisent cet espace avant celles d'origine rurale.

Néanmoins, les conflits les plus violents ont lieu entre Kosovars et Africains. En effet, les premiers n'acceptent ni de partager les dortoirs, ni de s'attabler avec les seconds. Ce n'est pas la peur qui alimente ces dissymétries, mais bien un racisme anti-noir que les Kosovars ne manquent pas d'exprimer, au point d'oublier, pendant un moment, leurs propres tensions pour s'unir contre les autres, ces Africains souvent dépourvus de soutien vu leur faible nombre. Manifestement, c'est le non-vouloir vivre ensemble avec l'autre qui crée, voire exacerbe, les tensions entre ces communautés censées partager les souffrances de l'exil et le drame de la guerre.

Il ressort des entretiens effectués avec les 90 personnes de notre échantillon que la régulation des conflits souterrains se fait au coup par coup à chaque apparition de dissymétries relationnelles et surtout grâce à l'intervention du personnel gérant l'abri. Réunions, discussions, négociations, menaces d'expulsion, concessions, aboutissent à des solutions «d'accommodation» pour sauvegarder la vie en commun. Ces accommodations correspondent à une force sourde de la vie, le règlement, qui vise à normer la coexistence dans des sous-espaces de fragile autonomie. C'est une complexe adaptation prise entre une empreinte humaine de liberté et un environnement de contraintes.

\section{Conclusion}

L'abri est abordé comme un espace d'enracinement et d'appropriation. Il a subi quelques transformations fonctionnelles, individuelles et collectives, adaptées à l'objectif de création d'un «chez soi», tout en restant conformes à la structure du lieu et à la surcharge humaine qu'il doit supporter. Il reflète des modes d'organisation interne, des tensions entre les occupants, des divergences opposant une conception fonctionnelle imposée par le règlement AGECAS-Protection Civile et des pratiques d'appropriation ou de ré-appropriation émanant des résidents. C'est précisément l'étude de ces pratiques qui a permis de révéler une dimension cachée de l'espace clos, devenu support de stratégies de réaménagement, de protection et d'inscription personnelle dans un lieu censé être a-personnel.

Passer l'essentiel de sa journée dans l'abri, n'y sortir que pour aller faire des courses, téléphoner à un proche ou aller rencontrer un ami, sont autant de facteurs poussant le requérant à s'investir davantage dans le réaménagement et la réorganisation de cet espace. En effet, les réfugiés n'avaient pas de contacts avec la population résidant autour de l'abri. A cela s'ajoute la non-fréquentation des lieux de resocialisation comme le lieu de travail, car l'exercice d'une activité professionnelle leur est strictement interdit par la loi, ou l'école pour les enfants, étant donné qu'ils sont arrivés en milieu d'année scolaire. De plus, ils estiment être mal perçus par la population environnante, qui parle d'ailleurs de Kosovars chaque fois qu'un délit est commis dans le quartier.

Par ailleurs, les Kosovars essaient de se retrouver, de recréer les solidarités secondaires basées sur les relations villageoises, claniques ou de voisinage grâce à l'espace de rencontre offert par l'Université Populaire Albanaise de Genève. Si cette dernière leur permet d'avoir des contacts, elle les «ghettoïse», puisque les Kosovars cherchent plus la compagnie de leurs «compatriotes» (comme ils le disent) qu'aller vers l'autre, même s'il est leur hôte.

En somme, les territorialités des requérants d'asile sont caractérisées par un double enfermement. L'enfermement dans un espace souterrain (l'abri) et l'enfermement dans des relations de proximité axées sur les logiques de liens (villageois, claniques, ethniques), logiques qui conditionnent la pratique de certains lieux comme chez les proches ou l'Université Populaire Albanaise de Genève.

\section{Bibliographie}

BAILly, A. (1986):L'espace à la croisée de l'exceptionnel et de l'universel: l'exemple des représentations territoriales. - In: La théorie de l'espace humain. Transformations globales et structures locales. - Paris: Craal- 
Fonds National de la Recherche Scientifique FNRS, Unesco: 228.

Bailly, A. (1991): Les concepts de la géographie humaine. - Paris: Masson.

Calhoun, J. B. (1962): Population density and social pathology. - In: Scientific American 206: 139-146.

Ferrier, J.-P. (1998): Le contrat géographique ou l'habitation durable des territoires. $-=$ Antée 2, Lausanne: Payot.

FischeR, G.-N. (1981): La psychosociologie de l'espace. - Paris: Presses Universitaires de France.

Fischer, G.-N. (1983): Le travail et son espace. De l'appropriation à l'aménagement. - Paris: Dunod.

LORENZ, K. (1970): Essais sur le comportement animal et humain. - Paris: Seuil.

Moles, A. \& E. Rohmer (1972): Psychologie de l'espace. - Paris: Castermann.

Moles, A. (1972 ): La perception de l'habitat. - Paris:

Editions Universitaires.

Résumé: Les pratiques spatiales des requérants d'asile dans les abris de la protection civile de Genève

Les requérants d'asile de Genève, installés dans les abris de la protection civile, ne sont pas restés des acteurs spatiaux passifs. Ils ont symboliquement transformé, artificiellement découpé les espaces abri afin de créer des coquilles personnelles les protégeant du regard des voisins et de leur empiétement. Leur stratégie, axée sur une organisation rationnelle de l'espace occupé, tient compte de l'enfermement et de l'entassement.

\section{Summary: The Spatial Behaviour of Asylum Seekers} in Geneva's Civil Defence Shelters

Asylum seekers in Geneva who have been put up in civil defence shelters have not remained inactive as spatial occupants. They have symbolically transformed the space allotted to them and have divided it artificially in order to create personal spheres which protect them from the neighbour's prying eyes or enquiring look. Their strategy, based on a rational organisation of the occupied space, takes the confinement and the crowdedness of their surroundings into account.
Zusammenfassung: Die raumbezogenen Verhaltensweisen von Asylsuchenden in Genfer Zivilschutzanlagen

Asylsuchende in Genf, welchen Wohnraum in Zivilschutzräumen zugewiesen wurde, sind in ihren raumbezogenen Verhaltensweisen nicht passiv geblieben. Sie haben die Schutzräume behelfsmässig verändert und künstlich abgetrennt, um persönliche Bereiche zu schaffen, die sie vor den Blicken der Nachbarn und deren Neugier schützen sollen. Ihre Strategie basiert auf einer rationellen Organisation des zur Verfügung stehenden Raumes und trägt der Beengtheit und dem Gedränge Rechnung.

Dr Karim Mouzoune, Université de Genève, Faculté des sciences économiques et sociales, 40 boulevard du Pont d'Arve, $\mathrm{CH}-1211$ Genève 4.

e-mail: mouzoune@geneva-link.ch 